\title{
Mechanism of Aqueous Carbon Dioxide Reduction by the Solvated Electron
}

\author{
Vladimir V. Rybkin* \\ Department of Chemistry, University of Zurich, Winterthurerstrasse 190, Zurich 805\%, \\ Switzerland \\ E-mail: vladimir.rybkin@chem.uzh.ch \\ Phone: +41 446354480
}




\begin{abstract}
Aqueous solvated electron, $e_{a q}^{-}$, a key species in radiation and plasma chemistry, can efficiently reduce $\mathrm{CO}_{2}$ in a potential green chemistry application. Here, the mechanism of this reaction is unravelled by condensed-phase Born-Oppenheimer molecular dynamics based on the correlated wave function and accurate DFT approximation. We introduce and apply the holistic protocol for solvated electron's reactions encompassing all relevant reaction stages starting from diffusion. The carbon dioxide reduction proceeds via a cavity intermediate, which is separated from the product, $\mathrm{CO}_{2}{ }^{-}$, by an energy barrier due to the bending of $\mathrm{CO}_{2}$ and the corresponding solvent reorganization energy. The formation of the intermediate is caused by solvated electron's diffusion, whereas the intermediate transformation to $\mathrm{CO}_{2}{ }^{-}$is triggered by solvent fluctuations. This picture of activation-controlled $e_{a q}^{-}$reaction is very different from both rapid barrierless electron transfer, and proton-coupled electron transfer, where key transformations are caused by proton migration.
\end{abstract}

\title{
Graphical TOC Entry
}

\begin{tabular}{|l|}
\hline TOC ENTRY REQUIRED \\
\hline
\end{tabular}

\section{Keywords}

solvated electrons, $\mathrm{CO}_{2}$ reduction, many-body electronic structure theory, molecular dynamics 
The bulk hydrated electron, $e_{a q}^{-}, 1,2$ is a key species in aqueous radiation ${ }^{3}$ and plasma chemistry. ${ }^{4}$ The structure of this non-standard species has long been elusive due to short life times and inaccessibility to direct structural methods. This opened opportunities for theory, although reliable modelling of $e_{a q}^{-}$is at least equally challenging as the corresponding experiments. An example of a controversy originated in different theoretical approaches is whether the hydrated electron occupies a cavity or not. ${ }^{5}$ Recent theoretical ${ }^{6-9}$ and experimental ${ }^{10}$ efforts have practically ruled out the non-cavity hypothesis setting standards for reliable modelling of the bulk hydrated electron.

The first method of choice is condensed-phase molecular dynamics (MD) based on the second-order Møller-Plesset theory (MP2). ${ }^{7,11}$ MP2 provides accurate description of liquid water, ${ }^{12}$ is free of delocalization error ${ }^{13}$ pernicious for the description of radicals and is, moreover, a strict ab initio method, i.e. is not based on empiricism and heuristic approximations. This comes at a price of extraordinary computational complexity, making each application exclusive. ${ }^{14}$ A computationally cheaper alternative is density functional theory (DFT) based on hybrid exchange-correlation functional with a tuned amount of exact exchange ${ }^{6}$ and non-local van der Waals correction. ${ }^{15}$

These methods can now be applied to the reactivity of $e_{a q}^{-} \cdot{ }^{16-19} \mathrm{In}$ addition to the requirements for modelling the solvated electron per se (accurate electronic electronic structure theory, dynamics, periodic boundary conditions) another challenge arises: preparation of realistic initial conditions. These should encompass the well-defined cavity (to ensure the solvated rather pre-solvated state $^{8}$ ) separated from the substrate to model the diffusion. The published theoretical work on the reactivity of the bulk solvated electron is based on simpler approaches lifting some of the aforementioned requirements. Some studies involve dynamic cluster models rather than the condensed-phase ones. ${ }^{20,21}$ Another approach is simulating substrates after electron attachment in clusters ${ }^{17,22}$ and in the condensed phase. ${ }^{23,24}$ Finally, a condensed-phase MD simulation of the reaction between acetamide and solvated electron in the condensed phase has been performed based on the simple generalized gradient ap- 
proximation (GGA) DFT, ${ }^{25}$ revealing a fast barrierless electron transfer. Despite the useful MD protocol, these results should be assessed critically: pure GGA DFT functionals suffer from the infamous delocalization error.

Here, we present condensed-phase ab initio MD simulations of $\mathrm{CO}_{2}$ reduction by $e_{a q}^{-}$ in high-temperature water at the appropriate levels of electronic structure theory: hybrid functional DFT and MP2. This reaction has been suggested for atmospheric carbon dioxide capture. ${ }^{26,27}$ In addition, anion radical $\mathrm{CO}_{2}{ }^{-}$is often applied as a strong reducing agent ${ }^{28}$ in plasma and radiation chemistry. The process is not diffusion controlled and has a barrier of ca. $16 \mathrm{~kJ} / \mathrm{mol}^{29}$ of the unknown nature. On the other hand, this barrier is small enough to be able to observe the reaction in real time without introducing the bias based on the hypotheses about the mechanism. Furthermore, interesting nuclear dynamics is expected due to the $\mathrm{CO}_{2}$ structure change from linear to bent upon electron attachment. ${ }^{30,31}$

Thus, aqueous $\mathrm{CO}_{2}$ reduction is a prototypical complex reaction of the hydrated electron. Its dynamic modelling based on accurate electronic structure methods will make a step forward in understanding reactivity of the exotic chemical species and establish methodology and set standards for further simulations.

Cluster analysis of geometric structures allowed to divide the frames into four main structure types: separate reactants, intermediate, transition state (TS) region and the product, $\mathrm{CO}_{2}{ }^{-}$, shown in Figure 1. Initially $\mathrm{CO}_{2}$ is randomly oriented with respect to the solvated electron's cavity as shown by the evolution of the carbon dioxide tilting angle (see Figures 2 and $2(\mathrm{c}))$. It is clearly seen from trajectory analysis that it is the solvated electron moving towards $\mathrm{CO}_{2}$ during the diffusion stage. This is opposite to what is observed for the reaction between $e_{a q}^{-}$and hydronium, ${ }^{20}$ but is to be expected based on the diffusion constant values: proton is a rare species more mobile than solvated electron in water due to the Grotthus mechanism. ${ }^{32}$

The intermediate is similar to the stable solvated electron's cavity (formed by either four or five water molecules) with one water molecule substituted with $\mathrm{CO}_{2}$ (Figure $1(\mathrm{~b})$ ). Unlike 
$\mathrm{H}_{2} \mathrm{O}$ it is not dipole oriented with the $\mathrm{CO}$ bonds towards the cavity, but tilted to the cavity surface at an angle of $100-120^{\circ}$, due to the non-polar character of the bonds (Figure 2(c)). Gyration radius remains the same as in the neat aqueous cavity (Figure 2(b)) and $\mathrm{CO}_{2}$ remains linear (Figure 2(c)), whereas $\mathrm{CO}_{2}$ remains linear. Transition region is characterized by the displacement $\mathrm{CO}_{2}$ towards the cavity center and the distortion of the nearly spherical spin density distribution, although the gyration radius remains unchanged (see Figures 1(c) and $2(\mathrm{~b}))$. Finally, the product, $\mathrm{CO}_{2}{ }^{-}$is bent, whereas spin density distribution exhibits characteristic nodal structure with significant spin polarization and small gyration radius (see Figures $1(\mathrm{~d})$ and 2(b)).

Cluster analysis assigns many structures with collapsed cavity and bent $\mathrm{CO}_{2}{ }^{-}$as belonging to the transition region (1.5 to $1.7 \mathrm{ps}$ in Figure 2). On the one hand it reflects the unsupervised character of the procedure (not based on chemical intuition). On the other hand, it highlights non-instantaneous solvent reorganization after the cavity collapse.

The qualitative picture of the reaction dynamics is summarized in equation (1) and Table 1 (see also the video in the ESI):

$$
\mathrm{e}^{-}+\mathrm{CO}_{2} \stackrel{\text { diffusion }}{\rightleftharpoons} \mathrm{e} \cdot . . \mathrm{CO}_{2}{ }^{-} \stackrel{\text { barrier }}{\rightleftharpoons}\left[\mathrm{e} \cdot . . \mathrm{CO}_{2}-\right]^{*} \longrightarrow \mathrm{CO}_{2}^{-}
$$

The intermediate shown in Figure 1(b) is formed via diffusion in all trajectories. Thus, we conclude that there is no barrier to the formation of the intermediate. Its lifetime can be up to 2 ps (limit of our time-scale) and is on average $0.5 \mathrm{ps.} \mathrm{In} \mathrm{half} \mathrm{of} \mathrm{the} \mathrm{trajectories}$ it converts to the products and it two DFT trajectories it falls apart to form the isolated $\mathrm{CO}_{2}$ and the solvated electron (Figure 1(a)). Thus, the intermediate must be a shallow local minimum.

The experimentally observed barrier is the one separating the intermediate and the products. Indeed, out of 15 observed attempts to form the product from the intermediate via the transition state (TS) region only 8 lead to the products in case of DFT and 2 of 5 in case of MP2, e.g. ca. 50\%. Unsuccessful crossing attempts are rapid as illustrated in Figure 2 (at 1 
ps in the graph), indicating that the corresponding structures are not stable, i.e. correspond to the TS region. This picture is in sharp contrast to the predicted barrierless reduction of amide ${ }^{25}$ being complete in less than 0.5 ps without formation of intermediates.

The nature of the barrier has to do with a large difference between vertical and adiabatic electron affinities of $\mathrm{CO}_{2} \cdot{ }^{33,34}$ In fact, free linear carbon dioxide does not bind an extra electron, whereas the anion is only stable in an angled conformation. ${ }^{35}$ Bending of $\mathrm{CO}_{2}$ requires significant energy and is followed by considerable solvent rearrangement.

Major structural changes in course of the reaction are marked with increase in the spin density distribution anisotropy (Figure 2(d)). Within the Born-Oppenheimer approximation, spin-density deformations are caused by solvent fluctuations. Thus, the latter trigger the main transformations: diffusion and barrier crossing. This is another difference between reduction mechanisms of $\mathrm{CO}_{2}$ and $\mathrm{H}^{+}$reduction. Electron transfer to the proton is launched by the "jumps" of the latter, ${ }^{20}$ rather than by solvent fluctuations.

In terms of Marcus theory, intermediate and transition state are strongly interacting correspond to the inner sphere electron transfer. ${ }^{36}$ In addition, such process is not described by Marcus theory of solvated electron reactions, as the latter assumes non-cavity polaron structure. ${ }^{37}$ Moreover, the cavity collapse after the reaction clearly goes beyond linear response description of reorganization energy. ${ }^{38}$

Here, a note must be made on performance of hybrid-functional DFT and MP2. Both methods provide qualitatively similar reaction picture. Due to the immense computational cost, the starting conditions for the MP2-driven MD have been selected from the DFT reactive trajectories to ensure the observation of at least a barrier-crossing attempt. The fact that only two of four lead to the products (the other two not being able to cross the barrier) provides evidence in favour of a larger barrier height of MP2 wrt. hybrid DFT. With $R T$ being $3 \mathrm{~kJ} / \mathrm{mol}$ at $350 \mathrm{~K}$, roughly every second encounter leading to a reaction (as observed with hybrid DFT) implies a much smaller barrier value than the experimentally found one of $16 \mathrm{~kJ} / \mathrm{mol} .{ }^{29}$ Thus, an apparently higher barrier brings MP2 in a better agreement 
with experiment, although the available statistics does not allow to make a less tentative conclusion.

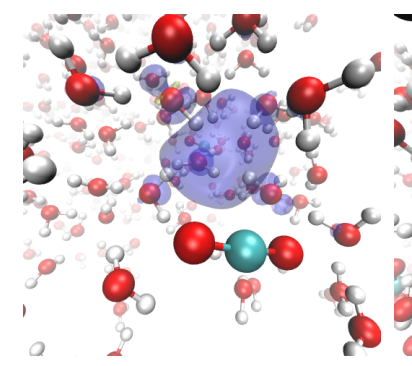

(a) Separated species: $e_{a q}^{-}$and $\mathrm{CO}_{2}$

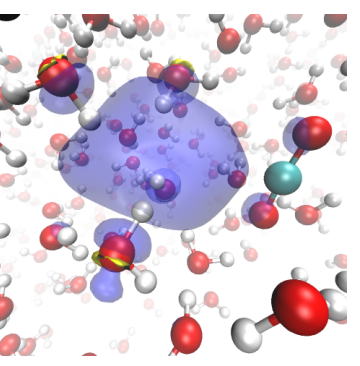

(b) Intermediate

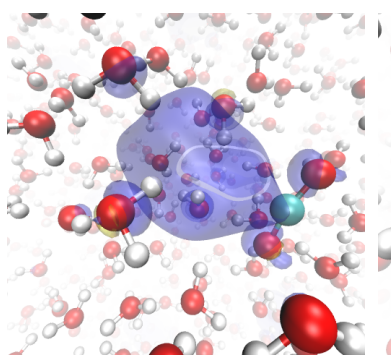

(c) Transition state region

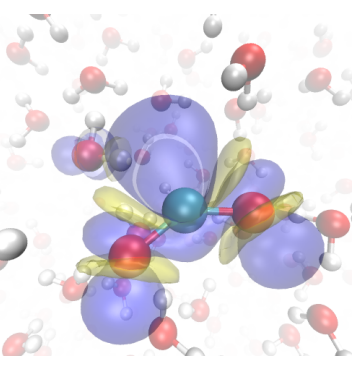

(d) Product: $\mathrm{CO}_{2}$

Figure 1: Representative structures of different stages of $\mathrm{CO}_{2}$ reduction by $e_{a q}^{-}$from an MP2 trajectory. Oxygen atoms are shown in red, hydrogen atoms in white, and carbon atoms in green. Spin density distribution isosurfaces are plotted in blue (positive) and yellow (negative) with isovalues of \pm 0.001 a.u.

Table 1: Trajectory outcomes. Number of unsuccessful attempts to cross the barrier is given in parentheses.

\begin{tabular}{lll}
\hline Method & Reactive & Non-reactive \\
\hline PBE50-rVV10 & $8(3)$ & $7(4)$ \\
MP2 & $2(1)$ & $2(2)$ \\
\hline
\end{tabular}

\section{Computational Methods}

\section{Electronic structure}

Production AIMD simulations were run based on DFT with hybrid exchange-correlation functional $^{39}$ and MP2 within spin-unrestricted formalism ${ }^{40}$ as implemented in the CP2K program. ${ }^{41}$ The core atomic levels were described with Goedecker-Teter-Hutter type pseudopotentials, ${ }^{42}$ whereas triple-zeta quality basis sets were used for valence shells. ${ }^{43,44}$

Hybrid functional DFT calculations were done with the modified hybrid PBE0 functional, ${ }^{45}$ where the amount of the exact exchange was increased to 50\% (PBE50), as the corresponding spin densities best approximate those from MP2. ${ }^{7}$ The non-local van der 


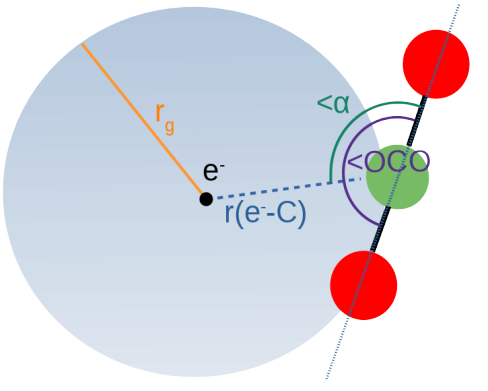

(a) Collective variables: $e^{-}-$the center of the spin density distribution (of an excess electron); $r_{g}$ - gyration radius of the spin density distribution; $r(e-C)$ - distance between $\mathrm{e}^{-}$and the carbon atom; $\angle \alpha$ - tilting angle of $\mathrm{CO}_{2} ; \angle O C O$ $\mathrm{CO}_{2}$ bending angle.

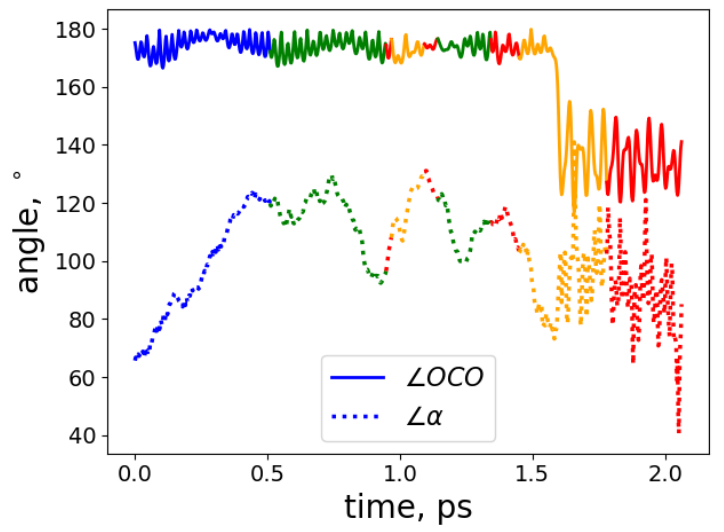

(c) $\angle O C O$ and $\angle \alpha$ vs. time.

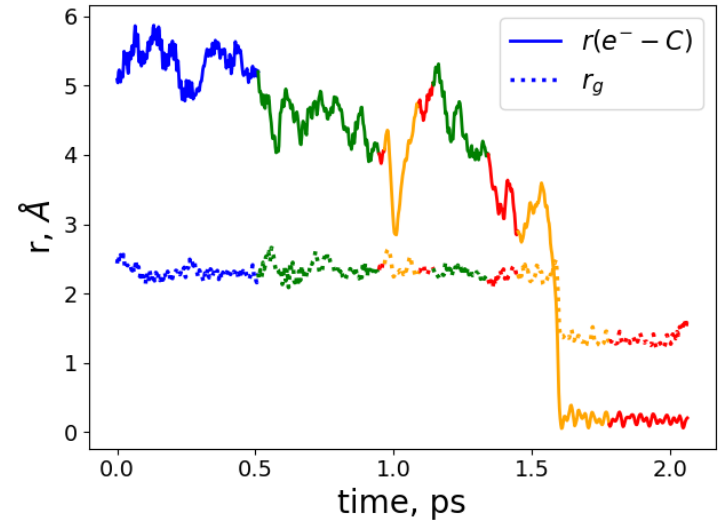

(b) $r\left(e^{-}-C\right)$ and $r_{g}$ vs. time.

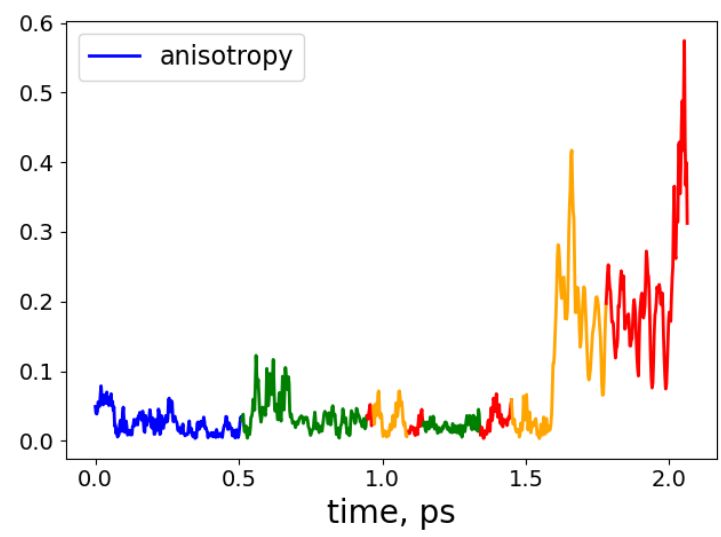

(d) Spin distribution anisotropy vs. time.

Figure 2: Time evolution of some collective variables along one of the reactive hybrid DFT trajectories. Colors correspond to the structure classification by cluster analysis: blue separate reactants (diffusion stage); green - intermediate; orange - transition state region; red - products. The formation of the intermediate is characterized by reorientation of the $\mathrm{CO}_{2}$ coming at a sharp angle $(\angle \alpha)$, but taking the position at the cavity with the obtuse tilting angle. The reaction completion is marked by the bending of the $\mathrm{CO}_{2}(\angle O C O)$, drop in gyration radius $\left(r_{g}\right)$ and transfer of the spin density to $\mathrm{CO}_{2}\left(r\left(e^{-}-C\right) \rightarrow 0\right)$ after 1.5 ps. An unsuccessful attempt to overcome the barrier takes place at ca. 1 ps. 
Waals correction was applied to improve the description of intermolecular interactions. ${ }^{15}$ This set-up is similar to the one successfully optimized for the solvated electron in works. ${ }^{6,8}$ More details are given in the ESI.

\section{Molecular dynamics and system preparation}

The system studied consists of 62 water molecules, a $\mathrm{CO}_{2}$ molecule and an excess electron. The periodic cell size is $13.9 \times 13.9 \times 13.9 \AA$, which corresponds to the experimental density of hot water at $350 \mathrm{~K}$ and normal pressure.

The system was prepared by equilibration (in an NVT ensemble at $350 \mathrm{~K}$ ) with a $\mathrm{Cl}^{-}$ instead of the excess electron to prevent the premature reaction, first with GGA DFT and then with hybrid functional DFT. Chloride has been shown to form a solvation shell of similar size and shape as the one of the hydrated electron. Initial conditions for the production trajectories have been randomly selected from the equilibrated trajectory, chloride being substituted with an excess electron ( $i$. e. chlorine nucleus removed). The initial distances between $e_{a q}^{-}$and $\mathrm{CO}_{2}$ varied from 4 to $6 \AA$.

A total of 15 production trajectories have been run with hybrid DFT (PBE50-rVV10) for either 2 ps or until the completion of the reaction in an NVE ensemble. Four production MP2 trajectories have been integrated. The intial conditions for the latter were taken from the

production runs with hybrid DFT, leading to the products. Due to extreme computational cost they were integrated either until the reaction completion, or after one or several attempts to cross the barrier. More details are given in the ESI.

\section{Structure classification}

Structures have been classified by cluster analysis based on dimensionality-reduced smooth overlap of atomic positions (SOAP) descriptor. ${ }^{46,47}$ The details are given in the ESI. 


\section{Acknowledgement}

This work has been supported by the Swiss National Science Foundation in the form of Ambizione grant No. PZ00P2_174227. This work was also supported by a grant from the Swiss National Supercomputing Centre (CSCS) under Project ID s976.

\section{Supporting Information Available}

This will usually read something like: "Experimental procedures and characterization data for all new compounds. The class will automatically add a sentence pointing to the information on-line:

\section{References}

(1) Herbert, J. M.; Coons, M. P. The Hydrated Electron. Annual Review of Physical Chemistry 2017, 68, 447-472, PMID: 28375692.

(2) Hart, E. J.; Boag, J. W. Absorption Spectrum of the Hydrated Electron in Water and in Aqueous Solutions. Journal of the American Chemical Society 1962, 84, 4090-4095.

(3) Garrett, B. C.; Dixon, D. A.; Camaioni, D. M.; Chipman, D. M.; Johnson, M. A.; Jonah, C. D.; Kimmel, G. A.; Miller, J. H.; Rescigno, T. N.; Rossky, P. J. et al. Role of Water in Electron-Initiated Processes and Radical Chemistry: Issues and Scientific Advances. Chemical Reviews 2005, 105, 355-390, PMID: 15720157.

(4) Rumbach, P.; Bartels, D. M.; Sankaran, R. M.; Go, D. B. The solvation of electrons by an atmospheric-pressure plasma. Nature Communications 2015, 6, 7248.

(5) Larsen, R. E.; Glover, W. J.; Schwartz, B. J. Does the Hydrated Electron Occupy a Cavity? Science 2010, 329, 65-69. 
(6) Ambrosio, F.; Miceli, G.; Pasquarello, A. Electronic levels of excess electrons in liquid water. The journal of physical chemistry letters 2017, 8, 2055-2059.

(7) Wilhelm, J.; VandeVondele, J.; Rybkin, V. V. Dynamics of the Bulk Hydrated Electron from Many-Body Wave-Function Theory. Angewandte Chemie International Edition 2019, 58, 3890-3893.

(8) Pizzochero, M.; Ambrosio, F.; Pasquarello, A. Picture of Wet Electron: A Localized Transient State in Liquid Water. Chemical Science 2019,

(9) Dasgupta, S.; Rana, B.; Herbert, J. M. Ab Initio Investigation of the Resonance Raman Spectrum of the Hydrated Electron. The Journal of Physical Chemistry B 2019, 123, 8074-8085, PMID: 31442044.

(10) Janik, I.; Lisovskaya, A.; Bartels, D. M. Partial Molar Volume of the Hydrated Electron. The Journal of Physical Chemistry Letters 2019, 10, 2220-2226.

(11) Møller, C.; Plesset, M. S. Note on an Approximation Treatment for Many-Electron Systems. Phys. Rev. 1934, 46, 618-622.

(12) Del Ben, M.; Schönherr, M.; Hutter, J.; VandeVondele, J. Bulk Liquid Water at Ambient Temperature and Pressure from MP2 Theory. The Journal of Physical Chemistry Letters 2013, 4, 3753-3759.

(13) Mori-Sánchez, P.; Cohen, A. J.; Yang, W. Localization and Delocalization Errors in Density Functional Theory and Implications for Band-Gap Prediction. Phys. Rev. Lett. 2008, 100, 146401.

(14) Rybkin, V. V. Sampling Potential Energy Surfaces in the Condensed Phase with ManyBody Electronic Structure Methods. Chemistry - A European Journal 2019, 26, 362368. 
(15) Vydrov, O. A.; Van Voorhis, T. Nonlocal van der Waals density functional: The simpler the better. The Journal of Chemical Physics 2010, 133, 244103.

(16) Anbar, M. Solvated Electron; Chapter 6, pp 55-81.

(17) Gu, J.; Leszczynski, J.; Schaefer, H. F. Interactions of Electrons with Bare and Hydrated Biomolecules: From Nucleic Acid Bases to DNA Segments. Chemical Reviews 2012, 112, 5603-5640, PMID: 22694487.

(18) Gordon, S.; Hart, E. J.; Matheson, M. S.; Rabani, J.; Thomas, J. K. Reactions of the hydrated electron. Discuss. Faraday Soc. 1963, 36, 193-205.

(19) Getoff, N. Possibilities on the radiation-induced incorporation of $\mathrm{CO} 2$ and $\mathrm{CO}$ into organic compounds. International Journal of Hydrogen Energy 1994, 19, 667 - 672.

(20) Marsalek, O.; Uhlig, F.; VandeVondele, J.; Jungwirth, P. Structure, Dynamics, and Reactivity of Hydrated Electrons by Ab Initio Molecular Dynamics. Accounts of Chemical Research 2012, 45, 23-32, PMID: 21899274.

(21) Kumar, A.; Adhikary, A.; Shamoun, L.; Sevilla, M. D. Do Solvated Electrons (eaq-) Reduce DNA Bases? A Gaussian 4 and Density Functional Theory-Molecular Dynamics Study. The Journal of Physical Chemistry B 2016, 120, 2115-2123, PMID: 26878197.

(22) Gu, J.; Xie, Y.; Schaefer, H. F. Near 0 eV Electrons Attach to Nucleotides. Journal of the American Chemical Society 2006, 128, 1250-1252, PMID: 16433542.

(23) Wu, X.; Gao, L.; Liu, J.; Yang, H.; Wang, S.; Bu, Y. Excess electron reactivity in amino acid aqueous solution revealed by ab initio molecular dynamics simulation: anioncentered localization and anion-relayed electron transfer dissociation. Phys. Chem. Chem. Phys. 2015, 17, 26854-26863.

(24) Wu, X.; Gao, L.; Liu, J.; Yang, H.; Wang, S.; Bu, Y. Excess electron reactivity in amino acid aqueous solution revealed by ab initio molecular dynamics simulation: anion- 
centered localization and anion-relayed electron transfer dissociation. Phys. Chem. Chem. Phys. 2015, 17, 26854-26863.

(25) Zhang, R.; Bu, Y. Bifurcate localization modes of excess electron in aqueous Ca2+ amide solution revealed by ab initio molecular dynamics simulation: towards hydrated electron versus hydrated amide anion. Phys. Chem. Chem. Phys. 2016, 18, 1886818879.

(26) Rumbach, P.; Xu, R.; Go, D. B. Electrochemical Production of Oxalate and Formate from CO2by Solvated Electrons Produced Using an Atmospheric-Pressure Plasma. Journal of The Electrochemical Society 2016, 163, F1157-F1161.

(27) Zhang, L.; Zhao, Z.-J.; Wang, T.; Gong, J. Nano-designed semiconductors for electroand photoelectro-catalytic conversion of carbon dioxide. Chem. Soc. Rev. 2018, 47, $5423-5443$.

(28) Armstrong, D. A.; Huie, R. E.; Koppenol, W. H.; Lymar, S. V.; Merényi, G.; Neta, P.; Ruscic, B.; Stanbury, D. M.; Steenken, S.; Wardman, P. Standard electrode potentials involving radicals in aqueous solution: inorganic radicals (IUPAC Technical Report). Pure and Applied Chemistry 2015, 87, 1139 - 1150.

(29) Lisovskaya, A.; Bartels, D. M. Reduction of CO2 by hydrated electrons in high temperature water. Radiation Physics and Chemistry 2019, 158, 61 - 63.

(30) Janik, I.; Tripathi, G. N. R. The nature of the CO2- radical anion in water. The Journal of Chemical Physics 2016, 144, 154307.

(31) Rybkin, V. V.; VandeVondele, J. Nuclear Quantum Effects on Aqueous Electron Attachment and Redox Properties. The Journal of Physical Chemistry Letters 2017, 8, 1424-1428, PMID: 28296416. 
(32) Herbert, J. M. Structure of the aqueous electron. Physical Chemistry Chemical Physics 2019, 21, 20538-20565.

(33) McNeill, A. S.; Dixon, D. A. Energetics of CO2- in Aqueous Solution. The Journal of Physical Chemistry A 2019, 123, 1243-1259, PMID: 30640468.

(34) Cheng, J.; VandeVondele, J. Calculation of Electrochemical Energy Levels in Water Using the Random Phase Approximation and a Double Hybrid Functional. Phys. Rev. Lett. 2016, 116, 086402.

(35) Gutsev, G. L.; Bartlett, R. J.; Compton, R. N. Electron affinities of CO2, OCS, and CS2. The Journal of Chemical Physics 1998, 108, 6756-6762.

(36) Marcus, R.; Sutin, N. Electron transfers in chemistry and biology. Biochimica et Biophysica Acta (BBA) - Reviews on Bioenergetics 1985, 811, 265 - 322.

(37) Marcus, R. A. Theory of Electron-Transfer Reaction Rates of Solvated Electrons. The Journal of Chemical Physics 1965, 43, 3477-3489.

(38) Bragg, A. E.; Cavanagh, M. C.; Schwartz, B. J. Linear Response Breakdown in Solvation Dynamics Induced by Atomic Electron-Transfer Reactions. Science 2008, 321, 18171822.

(39) Guidon, M.; Schiffmann, F.; Hutter, J.; VandeVondele, J. Ab initio molecular dynamics using hybrid density functionals. The Journal of Chemical Physics 2008, 128, 214104.

(40) Rybkin, V. V.; VandeVondele, J. Spin-unrestricted second-order Møller-Plesset (MP2) forces for the condensed phase: from molecular radicals to f-centers in solids. Journal of chemical theory and computation 2016, 12, 2214-2223.

(41) Kühne, T. D.; Iannuzzi, M.; Del Ben, M.; Rybkin, V. V.; Seewald, P.; Stein, F.; Laino, T.; Khaliullin, R. Z.; Schütt, O.; Schiffmann, F. et al. CP2K: An electronic 
structure and molecular dynamics software package - Quickstep: Efficient and accurate electronic structure calculations. The Journal of Chemical Physics 2020, 152, 194103.

(42) Goedecker, S.; Teter, M.; Hutter, J. Separable dual-space Gaussian pseudopotentials. Phys. Rev. B 1996, 54, 1703-1710.

(43) Del Ben, M.; Hutter, J.; VandeVondele, J. Electron Correlation in the Condensed Phase from a Resolution of Identity Approach Based on the Gaussian and Plane Waves Scheme. Journal of Chemical Theory and Computation 2013, 9, 2654-2671, PMID: 26583860.

(44) VandeVondele, J.; Hutter, J. Gaussian basis sets for accurate calculations on molecular systems in gas and condensed phases. The Journal of Chemical Physics 2007, 127, 114105.

(45) Adamo, C.; Barone, V. Toward reliable density functional methods without adjustable parameters: The PBE0 model. The Journal of Chemical Physics 1999, 110, 6158-6170.

(46) De, S.; Bartók, A. P.; Csányi, G.; Ceriotti, M. Comparing molecules and solids across structural and alchemical space. Phys. Chem. Chem. Phys. 2016, 18, 13754-13769.

(47) Himanen, L.; Jäger, M. O.; Morooka, E. V.; Canova, F. F.; Ranawat, Y. S.; Gao, D. Z.; Rinke, P.; Foster, A. S. DScribe: Library of descriptors for machine learning in materials science. Computer Physics Communications 2020, 247, 106949. 